\title{
P-Glycoprotein in proteoliposomes with low residual detergent The effects of cholesterol
}

\section{Journal Article}

Author(s):

Bucher, Karsten; Belli, Sara; Wunderli-Allenspach, Heidi; Krämer, Stefanie-Dorothea (D)

Publication date:

2007

Permanent link:

https://doi.org/10.3929/ethz-b-000003332

Rights / license:

$\underline{\text { In Copyright - Non-Commercial Use Permitted }}$

Originally published in:

Pharmaceutical Research 24(11), https://doi.org/10.1007/s11095-007-9326-0 


\title{
P-Glycoprotein in Proteoliposomes with Low Residual Detergent: The Effects of Cholesterol
}

\author{
Karsten Bucher, ${ }^{1}$ Sara Belli, ${ }^{1}$ Heidi Wunderli-Allenspach, ${ }^{1}$ and Stefanie D. Krämer ${ }^{1,2}$
}

Received February 14, 2007; accepted April 25, 2007; published online May 12, 2007

\begin{abstract}
Purpose. There is evidence that cholesterol affects the ATPase and transport functions of P-glycoprotein (P-gp). To study the influence of cholesterol on P-gp in a well defined lipid environment, we reconstituted $\mathrm{P}$-gp in egg phosphatidylcholine $(\mathrm{PhC})$ and $\mathrm{PhC} /$ cholesterol proteoliposomes with negligible residual amounts of detergents.

Materials and methods. P-gp proteoliposomes were prepared by continuous dialysis from micelles consisting of P-gp, lipids, sodium dodecyl sulfate and cholate. Basal and modulator-induced ATPase activities were studied in an established enzyme assay. Modulator affinities to P-gp and to the lipid bilayers were determined by equilibrium dialysis.

Results. In the absence of cholesterol the basal ATPase activity was six fold lower than in the presence of 20 or $40 \%$ cholesterol, and no P-gp binding and ATPase induction was detected for the tested modulators verapamil and progesterone. In proteoliposomes containing 20 and $40 \%$ cholesterol, respectively, the modulators showed significant P-gp binding and ATPase activation. The concentration of the modulators for half maximal activation of the ATPase was higher with $40 \%$ than with $20 \%$ cholesterol.

Conclusions. Cholesterol influences P-gp in three ways: (a) it enhances its basal ATPase activity, (b) it renders P-gp sensitive towards the modulators verapamil and progesterone and (c) it affects the modulator concentration at half maximal ATPase activation.
\end{abstract}

KEY WORDS: ATPase activity; binding; cholesterol; P-glycoprotein; verapamil.

\section{INTRODUCTION}

The multi-drug resistance protein $\mathrm{P}$-glycoprotein ( $\mathrm{P}$-gp) is an efflux transporter for a wide variety of drug compounds and cytotoxins. The $170 \mathrm{kDa}$ ATP binding cassette (ABC) protein contains 12 transmembrane domains and two cytosolic ATPbinding sites. It is encoded by the MDR1 (ABCB1) gene and is expressed under physiological conditions in tissues with excretory functions, such as the small intestine, liver and kidney, and at blood-tissue barriers such as the blood-brain barrier, blood-testis barrier and blood-placenta barrier. It is typically but not exclusively localized in the apical plasma membrane domain of P-gp expressing cells (1). P-gp gets frequently upregulated in tumor cells upon treatment with

\footnotetext{
${ }^{1}$ Department of Chemistry and Applied Biosciences, Institute of Pharmaceutical Sciences, ETH Zurich, Wolfgang-Pauli-Strasse 10, 8093, Zurich, Switzerland.

${ }^{2}$ To whom correspondence should be addressed. (e-mail: skraemer@ pharma.ethz.ch)

ABBREVIATIONS: CHAPS，3-[(3-cholamidopropyl)dimethylammonio]propanesulfonate; DTT, Dithiothreitol; DMPC, Di-myristoyl phosphatidylcholine; DPPC, Di-palmitoyl phosphatidylcholine; PBS, Phosphate buffered saline; TNE, Tris/ $\mathrm{NaCl} /$ EDTA buffer; P-gp, P-glycoprotein; PhC, Phosphatidylcholine; SDS, Sodium dodecyl sulfate; SUBS, Standardized universal buffer solution.
}

cytotoxic drugs, causing multi-drug resistance by expelling a wide palette of chemotherapeutics from these cells. For reviews see (1-3).

The transported substrates have no common structural features which would point to a well defined binding pocket in the transporter and which would allow to clearly distinguish them from non-substrates. As P-gp substrates are relatively lipophilic, it is assumed that the binding to the transporter occurs from within the lipid bilayer (2). Several studies on drug-P-gp interactions give indeed evidence for drug binding sites within the transmembrane domains of the protein (2,4-6). This is in line with the observations that the transport and ATPase activities of P-gp are strongly dependent on the lipid environment (7). P-gp may contain various binding sites for different compounds or display one common binding pocket of high flexibility to enable an induced fit mechanism (8). In addition to the model of direct drug binding to the transporter, indirect drug/P-gp interactions via the lipid bilayer or a $\mathrm{pH}$ gradient were discussed (9).

P-gp hydrolyses ATP in the absence of known substrates when reconstituted in a lipid bilayer environment; this is defined as the basal ATPase activity. The ATPase activity is significantly enhanced in the presence of many transported substrates and of so called modulators. The latter activate the P-gp ATPase activity without undergoing a significant P-gp related net transport. This phenomenon may occur for compounds which are translocated by P-gp but have a high 
passive permeation rate across the membrane, exceeding the transport efficiency of the protein (10).

$\mathrm{P}$-gp is enriched in detergent-insoluble membrane domains, the so called rafts, which contain relatively high amounts of cholesterol and sphingolipids (11-13). Cholesterol plays a crucial role for the function of P-gp as an ATPase and a transport system. In cell culture, the sterol controls P-gp functions and P-gp localization (11-18). In general, the ATPase activity of P-gp decreased upon depletion of cells from cholesterol and increased upon repletion or saturation of the cells with cholesterol.

It is still not known how the sterol influences the P-gp ATPase and transport functions (1). Cholesterol may be a substrate of $\mathrm{P}$-gp transport and therefore increase the ATPase activity in a similar manner as many known P-gp substrates. This is supported by several studies (19). However, the direction of the transport is not clear. Transport has been described as cell influx or translocation from the exoplasmic to the cytoplasmic membrane leaflet (20), as well as from the cytoplasmic to the exoplasmic leaflet (18).

Alternatively, cholesterol could directly interact with P-gp, enhancing its ATPase activity, without being transported. A third possibility is given by the influence of cholesterol on the characteristics of a lipid bilayer $(15,21)$. The sterol could thus indirectly affect the function and activity of P-gp via the membrane properties.

That cholesterol affects the ATPase activity of P-gp has not only been shown with P-gp expressing cells but also with proteoliposomes, i.e., after P-gp reconstitution in the lipid bilayer of lipid vesicles. Proteoliposomes have the advantage over whole cells that the transporter can be studied as an isolated protein. Indirect effects by a cellular response can be excluded. A great part of our current knowledge about the influence of the lipid environment on the efflux transporter $\mathrm{P}$-gp comes from excellent work with P-gp proteoliposomes $(7,14,22-30)$.

The work with reconstituted P-gp is hampered by the fact that most systems contain considerable amounts of residual detergents. The latter generally affect the activity of the transporter (31). In addition, mild detergents such as CHAPS do not completely delipidate the protein upon solubilization. The remaining lipids (about 50 phospholipid molecules per P-gp molecule) could affect the activity and function of the reconstituted transporter (32). We therefore established a method to prepare P-gp proteoliposomes with negligible amounts of residual detergents and lipids from the source membrane. This was achieved by the use of the strong detergents sodium dodecyl sulfate (SDS) and cholate for the solubilization and reconstitution of $\mathrm{P}$-gp into egg phosphatidylcholine $(\mathrm{PhC})$ and $\mathrm{PhC} /$ cholesterol proteoliposomes. Both detergents are quantitatively removable by dialysis due to their relatively high critical micelle concentration. The protocol was used to study the influence of cholesterol on the basal and modulator-induced ATPase activity of P-gp and on the binding of the modulators to the transporter. The investigated modulators were verapamil and progesterone.

The basal P-gp ATPase activity was about six times lower in the absence of cholesterol than in the presence of 20 or $40 \%$ ( $\mathrm{mol} / \mathrm{mol}$ total lipid) of the sterol. This low activity remained unchanged upon addition of the studied modulators and no specific P-gp binding of the tested compounds was observed. In proteoliposomes containing 20 and $40 \%$ cholesterol, respectively, the sterol not only enhanced the basal ATPase activity in the absence of the tested modulators, but also enabled P-gp binding and further ATPase activation by the modulators. Finally, the modulator concentrations at half maximal ATPase activation were higher at $40 \%$ than at $20 \%$ cholesterol. Based on these findings we put forward a new model for the functional interaction between cholesterol and P-gp.

\section{MATERIALS AND METHODS}

\section{Chemicals}

[N-methyl- $\left.{ }^{3} \mathrm{H}\right]$-verapamil $(3.145 \mathrm{TBq} / \mathrm{mmol}$, \# NET-810), $\left[1,2,6,7-{ }^{3} \mathrm{H}(\mathrm{N})\right]$-progesterone (3.77 $\mathrm{TBq} / \mathrm{mmol}$, \# NET 381) and $\left[2,4-{ }^{3} \mathrm{H}\right]$-cholic acid $(560 \mathrm{GBq} / \mathrm{mmol}$, \# CUS55054000MC) were purchased from Perkin Elmer Life Sciences (NEN, Boston MA, USA) and sodium dodecyl-1- ${ }^{14} \mathrm{C}$-sulfate $(2.035$ GBq/mmol, \# L-2656) from Sigma (St. Louis MO, USA). Adenosine triphosphate, di-sodium (ATP) \#A2383, orthovanadate, sodium \#S6508, progesterone \#P0130, and ( \pm )-verapamil \#V4629 were supplied by Sigma (Buchs, Switzerland), dithiothreitol (DTT) \#43815, NADH \#43420, phosphoenolpyruvate \#79418, pyruvate kinase \#83330, sodium dodecylsulfate (SDS) and 3-[(3-cholamidopropyl)dimethylammonio]propanesulfonate (CHAPS) were from Fluka (Buchs, Switzerland). Lactate dehydrogenase \#127876, DNAse I and the protease inhibitor cocktail Complete ${ }^{\circledR}$ were supplied by Roche Diagnostics (Basel, Switzerland). All other chemicals were of analytical grade.

\section{Cell Cultures and Antibodies}

P388/ADR cells were obtained from the National Cancer Institute (Frederick, MD, USA). Cells were grown in suspension in RPMI1640 medium (Invitrogen Corp., Basel, Switzerland) containing $10 \%$ heat inactivated fetal bovine serum and split 1:10 every 3-4 days. The monoclonal mouse antihuman P-gp antibody C219 was supplied by DAKO Corp. (DakaCytomation, Carpinteria, CA, USA).

\section{Purification of P-glycoprotein}

About four $10^{8} \mathrm{P} 388 / \mathrm{ADR}$ cells suspended in $2 \mathrm{ml} \mathrm{PBS}$, pH $7.0\left(50 \quad \mathrm{mM} \mathrm{Na} \mathrm{HPO}_{4} / \mathrm{NaH}_{2} \mathrm{PO}_{4}, 130 \mathrm{mM} \mathrm{NaCl}\right)$ containing $10 \mathrm{mM} \mathrm{MgCl}_{2}, 1 \mathrm{mM}$ dithiothreitol (DTT), 0.4 $\mathrm{mg} / \mathrm{ml}$ DNAse I and protease inhibitor cocktail Complete ${ }^{\circledR}$ were homogenized with 50 strokes of a Dounce homogenizer at $1,000 \mathrm{rpm}$. The membrane fragments were collected by centrifugation at $200,000 \mathrm{~g}$ for $1 \mathrm{~h}$ at $4^{\circ} \mathrm{C}$. The pellet containing the membrane fragments was solubilized in $2 \mathrm{ml}$ $50 \mathrm{mM} \mathrm{Na} 2 \mathrm{HPO}_{4} / \mathrm{NaH}_{2} \mathrm{PO}_{4}, \mathrm{pH} 7.4$, containing $1 \mathrm{mM}$ DTT in $2 \%(\mathrm{~m} / \mathrm{v})$ SDS. After solubilisation the preparation was diluted with $50 \mathrm{mM} \mathrm{Na} \mathrm{HPO}_{4} / \mathrm{NaH}_{2} \mathrm{PO}_{4}, \mathrm{pH}$ 7.0, containing $1 \mathrm{mM}$ DTT to $1 \%$ SDS. P-gp was purified from the solubilized membrane fragments by HPLC according to (33) with some modifications. Briefly, about $4 \mathrm{mg}$ protein were injected on a hydroxyapatite cartridge $\left(\right.$ Econo-Pac ${ }^{(}$ CHT-II Biorad, Hercules CA, USA) and eluted with a 
sodium phosphate gradient (50 to $650 \mathrm{mM}$ phosphate), $\mathrm{pH}$ 7.0, containing $0.5 \%$ SDS and $1 \mathrm{mM}$ DTT. The buffer components were incubated at $40^{\circ} \mathrm{C}$ and the cartridge was heated to $30^{\circ} \mathrm{C}$ to prevent SDS precipitation. P-gp eluted at about $530 \mathrm{mM}$ phosphate and was concentrated with Vivaspin 15R Hydrosart $\left.{ }^{(}\right)$concentrators (mw cutoff 100,000, Sartorius, Germany) before being stored at $-80^{\circ} \mathrm{C}$. The concentrates contained $3-5 \mathrm{mg}$ protein per $10^{8}$ cells starting material. This purification protocol results in $\mathrm{P}$-gp which is virtually free of lipids (33).

\section{Reconstitution of P-glycoprotein}

Protein-free liposomes of the final lipid composition were prepared by extrusion in PBS, pH 7.4 (34). The liposomes were subsequently dissolved by $30 \mathrm{~min}$ incubation with sodium cholate at $60^{\circ} \mathrm{C}$ at a lipid/detergent molar ratio between 0.5 ( $\mathrm{PhC} /$ cholesterol liposomes) and 0.72 ( $\mathrm{PhC}$ liposomes) to form micelles. Complete solubilization was confirmed by dynamic light scattering with a Zetasizer 3000 HSA (Malvern Instruments, Malvern, UK).

Purified P-gp (between 1.5 and $5 \mathrm{mg}$ ) in phosphate buffer containing $\sim 0.25 \%$ SDS (see above) was added to the micelles (between 15 and $50 \mathrm{mg}$ lipids) at room temperature in a total volume between 6 and $10 \mathrm{ml}$. All subsequent steps were performed at room temperature to prevent SDS precipitation. After equilibration over night under argon the protein/lipid micelles were continuously dialysed against 201 of PBS containing $1 \mathrm{mM}$ DTT and Complete ${ }^{\circledR}, \mathrm{pH} 7.4$, by means of a Liposomat (Diachema, Munich, Germany) equipped with a high permeability preconditioned cellulose membrane with an mw cutoff of 10,000 and a size of $18 \times 18 \mathrm{~cm}^{2}$ (Diachema). Flow rates were $0.6 \mathrm{ml} / \mathrm{min}$ on the micelle side and $2.5 \mathrm{ml} / \mathrm{min}$ on the buffer side (35). Dialysis membranes were exchanged 2-3 times during the dialysis.

To monitor detergent removal, ${ }^{3} \mathrm{H}$-cholate and ${ }^{14} \mathrm{C}$-SDS $(20,000 \mathrm{~Bq} / \mathrm{ml}$ each) were added to the micelles together with the purified P-gp. Samples were taken during the dialysis to confirm the stability of the protein by SDS-polyacrylamide gel electrophoresis (SDS-PAGE) and immunoblotting as well as to determine the ATPase activity and the detergent concentration. After $96 \mathrm{~h}$ of continuous dialysis the proteoliposomes were immediately used for the ATPase assays. The protein content of the final proteoliposomes was determined with the DC protein assay kit from Biorad with bovine serum albumin as standard. $\mathrm{PhC}$ and cholesterol concentrations were determined by HPLC as described (36).

\section{Density Centrifugation and SDS-PAGE}

Proteoliposomes $(2 \mathrm{ml})$ were transferred to an equal volume of $80 \%(\mathrm{w} / \mathrm{v})$ sucrose in TNE $(25 \mathrm{mM}$ Tris, $150 \mathrm{mM}$ $\mathrm{NaCl}, 5 \mathrm{mM}$ EDTA, and Complete ${ }^{\circledR}, \mathrm{pH}$ 7.4) into a centrifuge tube and overlaid with $2 \mathrm{ml}$ each of $30 \%, 20 \%$, $10 \%$ and $1.5 \mathrm{ml}$ of $5 \%$ sucrose in TNE. After centrifugation at $200,000 \times \mathrm{g}$ for $19 \mathrm{~h}$ at $4^{\circ} \mathrm{C}$, proteoliposomes were visible as a turbid band in the upper part of the tube corresponding to a density range between 1.03 and $1.05 \mathrm{~g} / \mathrm{cm}^{3}$ as determined from the refractive indices. Fractions of $1 \mathrm{ml}$ were collected and the P-gp distribution was studied by SDS-PAGE, 7.5\%, and immunoblotting with the anti-P-gp-antibody C219.

\section{ATPase Kinetic Assay}

The ATPase activities ( $V_{\text {app }}$ ) of the proteoliposomes were determined at $37^{\circ} \mathrm{C}$, pH 7.4, in 96 well plates (Corning, flat bottom, black, \#3915, Corning, Acton, MA, USA) with a Synergy HT plate reader (BioTek, Winooski VT, USA) as described (12). In brief, the ATP consumption was quantified via an enzyme cascade resulting in NADH oxidation and ATP recycling. The concentration of ATP was $9 \mathrm{mM}$ (12). The concentration-time profile of NADH was determined from its fluorescence every $75 \mathrm{~s}$. The protein content per well was between 1 and $6 \mu \mathrm{g}$, the total sample volume per well $200 \mu \mathrm{l}$.

The $V_{\text {app }}$ /concentration profiles obtained with verapamil and progesterone were fitted with Eq. 1, excluding the modulator concentrations $\geq 100 \mu \mathrm{M}$, at which an unspecific inhibition occurred (12).

$$
V_{\text {app }}=V_{\text {basal }}+\frac{V^{\prime}}{1+\frac{K}{x}}
$$

In Eq. $1, V^{\prime}$ denotes the difference between the activity at a modulator concentration $x \rightarrow \infty$ (neglecting the inhibitory effect at high concentrations) and the basal activity ( $\left.V_{\text {basal }}\right)$ in the absence of modulator. $K$ is the concentration of modulator at half $V^{\prime}$. For clarity, the data are shown in plots with a logarithmic concentration scale. Data were fitted using the Microsoft Excel Solver tool.

To test the influence of CHAPS, proteoliposomes were incubated for $24 \mathrm{~h}$ with $400 \mu \mathrm{M}$ of the detergent at $37^{\circ} \mathrm{C}$ before they were diluted as stated above to determine the ATPase activity at different verapamil concentrations. The final CHAPS concentration in the assay was $28 \mu \mathrm{M}$.

\section{Binding Affinity to P-glycoprotein}

The binding of verapamil and progesterone to $\mathrm{P}$-gp proteoliposomes was determined at $37^{\circ} \mathrm{C}$ by equilibrium dialysis with $0.2 \mathrm{ml}$ dialysis cells and cellulose membranes (mw cutoff 10,000, Dianorm, Munich, Germany). Total drug concentrations were between $10^{-9}$ and $10^{-4} \mathrm{M}$, containing $1,600 \mathrm{~Bq} / \mathrm{ml}{ }^{3} \mathrm{H}$-verapamil and 2,700 $\mathrm{Bq}{ }^{3} \mathrm{H}$-progesterone, respectively. The protein concentration was between 0.1 and $0.5 \mathrm{mg} / \mathrm{ml}$. Dialysis was performed in PBS, $\mathrm{pH} 7.4$, containing $200 \mu \mathrm{M}$ sodium vanadate, $10 \mathrm{mM} \mathrm{MgCl}_{2}$ and $1.2 \mathrm{mM} \mathrm{ATP}$ (37). The dialyzed samples contained in addition $\sim 0.5 \%$ ethanol originating from the stock solutions of the modulators.

The dialysis was terminated after $5 \mathrm{~h}$ (38) and verapamil and progesterone, respectively, were quantified in both cell chambers by liquid scintillation counting. The protein and lipid concentrations in the respective chambers were determined as described above.

The binding data were transformed to Scatchard plots and the data obtained with the $\mathrm{PhC} /$ cholesterol proteoliposomes were further analyzed by non-linear curve fitting according to Eq. 2

$$
\frac{r}{C_{u}}=\frac{n_{1} \times K_{\mathrm{a} 1}}{1+K_{\mathrm{a} 1} \times C_{u}}+\frac{n_{2} \times K_{\mathrm{a} 2}}{1+K_{\mathrm{a} 2} \times C_{u}}
$$

where $r$ is the ratio of bound compound [M] and total protein [M] (mw 170,000, assuming $100 \% \mathrm{P}-\mathrm{gp}$ ), $C_{u}$ is the unbound 
drug concentration $[\mathrm{M}], n_{i}$ is the maximal number of compound molecules bound to the binding site(s) of class $i$ [-] and $K_{a i}\left[\mathrm{M}^{-1}\right]$ is the association constant between a drug molecule and a binding site of class $i$.

\section{Membrane Affinity}

The affinity of ${ }^{3} \mathrm{H}$-verapamil and ${ }^{3} \mathrm{H}$-progesterone to liposomal membranes was determined by equilibrium dialysis at $37^{\circ} \mathrm{C}(38)$. The $\mathrm{pH}$ was adjusted with a standardized universal buffer solution (SUBS, (38)) as indicated. The drug concentrations were $10^{-7} \mathrm{M}$ and $\mathrm{PhC}$ concentrations were about $1 \mathrm{mg} / \mathrm{ml}$. Distribution coefficients $(D)$ were calculated according to Eq. 3 (34)

$$
D=\left(C_{L B}-C_{B}\right) \times v_{L B} /\left(C_{B} \times v_{L}\right)+1
$$

where $C_{L B}$ is the molar solute concentration in the liposomecontaining chamber and $C_{B}$ in the buffer chamber. The symbol $v_{L B}$ denotes the sample volume of the liposome suspension, $v_{L}$ the volume of the lipophilic phase, i.e., the lipid bilayers. This volume was calculated from the $\mathrm{PhC}$ concentrations in the dialysis cells, which were determined by HPLC (see above), and a density of $1.0 \mathrm{~g} / \mathrm{ml}$ for the lipids. The $\mathrm{pH}$-dependent distribution of ${ }^{3} \mathrm{H}$-verapamil between the lipid bilayer and the aqueous phase was fitted according to Eq. 4 (34):

$$
D=\alpha_{N} \times P^{N}+\alpha_{I} \times P^{I}
$$

where $\alpha_{N}$ and $\alpha_{I}$ are the molar fractions of neutral and ionized verapamil, respectively $\left(\mathrm{pK}_{\mathrm{a}} 8.9\right)$, and $P^{N}$ and $P^{I}$ are the partition coefficients of the two ionization species.

\section{RESULTS}

\section{Characterization of P-gp Proteoliposomes}

P-gp was purified from $\mathrm{P} 388 / \mathrm{ADR}$ cells and reconstituted into $\mathrm{PhC}, \mathrm{PhC} / \mathrm{cholesterol} 80 / 20(\mathrm{~mol} / \mathrm{mol})$ and $\mathrm{PhC} / \mathrm{choles}-$ terol 60/40 liposomes, respectively, as described under Materials and Methods. The final protein concentration of a typical preparation was $0.2 \mathrm{mg} / \mathrm{ml}$. The protein to lipid ratio (w/w) after reconstitution was between 0.10 and 0.13 , corresponding to molar ratios between $4.5 \times 10^{-4}$ and $5.9 \times 10^{-4}$.

As detergents can significantly affect the ATPase activity of P-gp $(31,39)$, the detergent concentrations in the proteoliposomes were determined with radio-labeled SDS and cholate (see Materials and Methods). The residual concentrations were below the detection limit. The corresponding lipid/detergent and protein/detergent ratios are depicted in Table I. The respective detergent concentrations had no influence on the ATPase activity of rafts from P388/ADR cells (12), which display high P-gp ATPase activity (data not shown).

To test whether P-gp was stable during the $96 \mathrm{~h}$ of dialysis needed to reach the low detergent concentrations, samples were analyzed by SDS-PAGE and immunoblotting after 9, 24, 48 and $72 \mathrm{~h}$ and at the end of the dialysis, i.e., after 96 h. As shown in Fig. 1a, no decomposition products were visible on the blot and the amount of P-gp was similar at all tested time points. The ATPase activity after 19 and $48 \mathrm{~h}$ of dialysis was 67 and $82 \%$, respectively, of the final activity reached after $96 \mathrm{~h}$, indicating the gain in activity by the removal of the detergents (data not shown). In addition to the dialysate all eluted fractions from the hydroxyapatite cartridge were checked for P-gp decomposition products by SDS-PAGE and immunoblot. No such products were detected in several purification runs (data not shown).

To confirm the successful reconstitution of P-gp, a density gradient centrifugation of the proteolioposome preparation was performed and the density fractions were analyzed by SDS-PAGE and immunoblotting. As shown in Fig. 1b there was complete reconstitution indicated by the localization of $\mathrm{P}-\mathrm{gp}$ in the lipid containing turbid low density fractions (fractions $1-4,1.03-1.05 \mathrm{~g} / \mathrm{cm}^{3}$ ). No P-gp was detected in the high density fractions (fractions 5-9) where soluble or aggregated proteins would accumulate. This result was independent of the lipid composition.

The final P-gp proteoliposomes displayed a basal ATPase activity between 16.6 and $101.0 \mathrm{nmol}$ per min per mg protein depending on the lipid composition of the proteoliposomes (see next section). To verify that the observed ATPase activity originated from P-gp, ATPase activities were determined at different concentrations of the monoclonal P-gp antibody C219, which binds to both nucleotide binding regions of $\mathrm{P}$-gp and has been shown to inhibit its ATPase activity (40). C219 reduced the basal activity of the proteoliposomes to $(15.0 \pm 2.6) \%$ at $5 \mu \mathrm{g}$ antibody and $5 \mu \mathrm{g}$ P-gp per $\mathrm{ml}$. This was independent of the lipid composition. When the proteoliposomes were incubated with $400 \mu \mathrm{M}$ sodium orthovanadate, an unspecific ATPase inhibitor $(12,41)$, the ATPase activity was decreased to a similar residual activity of $(12.4 \pm 3.7) \%$ in all proteoliposomes.

The average size and size distribution of the proteoliposomes were characterized by dynamic light scattering with a Zetasizer 3000 HSA (Malvern Instruments, Malvern, UK). All proteoliposome preparations showed an average mean hydrodynamic diameter between 130 and $150 \mathrm{~nm}$ and a polydispersity index $<0.4$ corresponding to a size variance of $<63 \%$ assuming a monomodal distribution. No signs of aggregates were observed in the correlogram.

\begin{tabular}{|c|c|c|c|c|c|}
\hline \multirow{2}{*}{ Detergent } & \multirow{2}{*}{$\begin{array}{l}\text { Concentration } \\
{[\mathrm{M}]}\end{array}$} & \multicolumn{2}{|c|}{ Detergent/Protein Ratio } & \multicolumn{2}{|c|}{ Detergent/Lipid Ratio } \\
\hline & & {$[\mathrm{mg} / \mathrm{mg}]$} & {$[\mathrm{mol} / \mathrm{mol}]^{a}$} & {$[\mathrm{mg} / \mathrm{mg}]$} & {$[\mathrm{mol} / \mathrm{mol}]^{a}$} \\
\hline SDS & $<1.7 \times 10^{-6}$ & $<2 \times 10^{-3}$ & $<1.4$ & $<3 \times 10^{-4}$ & $\begin{array}{l}<7 \times 10^{-4} \\
<2 \times 10^{-4}\end{array}$ \\
\hline Cholate & $<4.0 \times 10^{-7}$ & $<8 \times 10^{-4}$ & $<0.3$ & $<9 \times 10^{-5}$ & $<2 \times 10^{-4}$ \\
\hline
\end{tabular}

Table I. Residual Detergent in P-gp Proteoliposomes

The protein concentration was $0.21 \mathrm{mg} / \mathrm{ml}$, the lipid concentration $1.8 \mathrm{mg} / \mathrm{ml}$.

${ }^{a}$ Calculated with a molecular weight of 170,000 for P-gp and 780 for egg PhC, respectively. 
Influence of Cholesterol on the Basal and Drug-induced ATPase Activity of Reconstituted P-gp

Cholesterol significantly influenced the basal ATPase activity of the proteoliposomes. In the absence of cholesterol (PhC proteoliposomes) it was $(16.6 \pm 2.1) \mathrm{nmol} \times \mathrm{min}^{-1} \times \mathrm{mg}^{-1}$ $(n=36)$ but reached $(101.0 \pm 8.1) \mathrm{nmol} \times \mathrm{min}^{-1} \times \mathrm{mg}^{-1}$ $(n=36)$ in proteoliposomes containing $20 \%$ cholesterol and $(86 \pm 20) \mathrm{nmol} \times \mathrm{min}^{-1} \times \mathrm{mg}^{-1} \quad(n=54)$ in proteoliposomes containing $40 \%$ cholesterol, respectively (Fig. $2 \mathrm{a}$ ).

Next, we investigated whether cholesterol had an effect on the ATPase activity in the presence of P-gp modulators. Verapamil and progesterone are known to enhance the

a

\section{Dialysis time [h]}
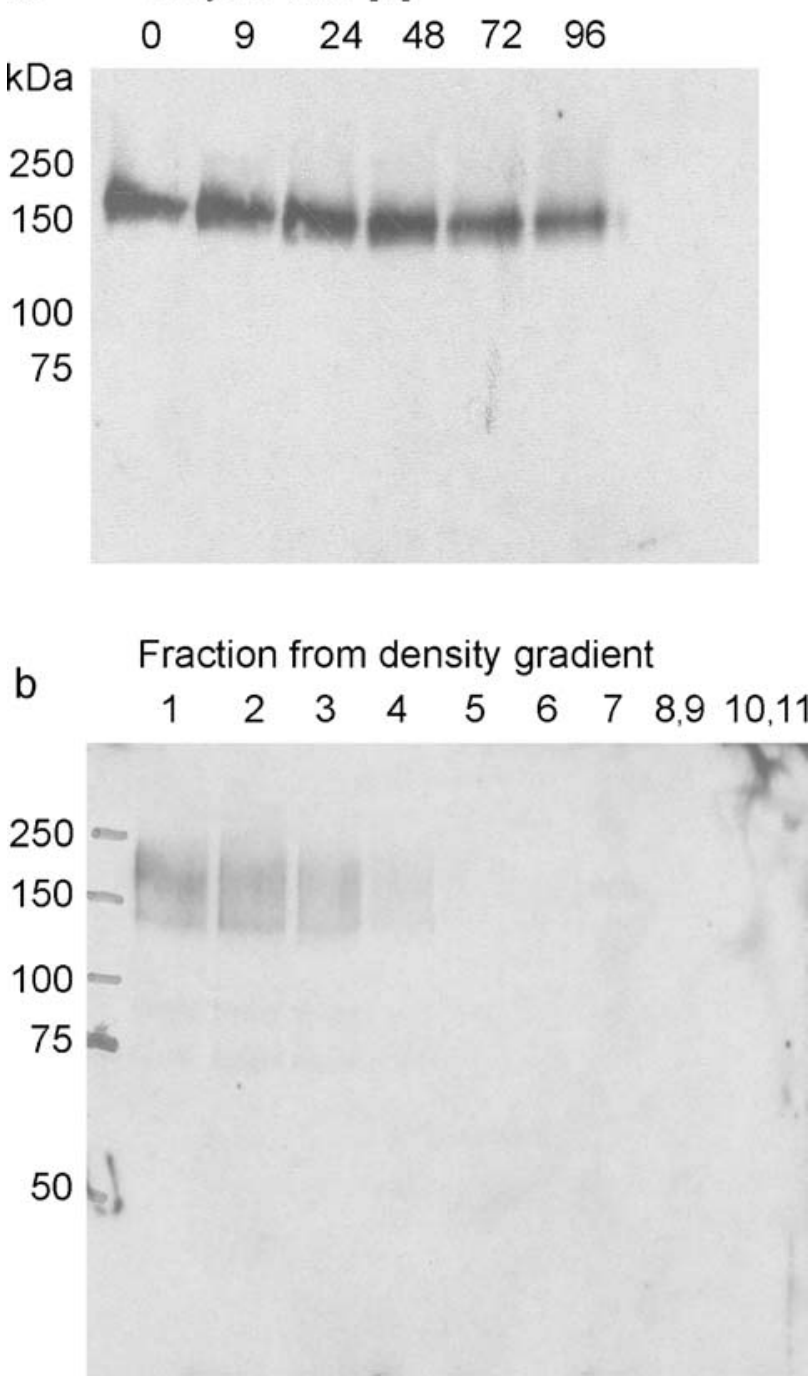

Fig. 1. Confirmation of P-gp reconstitution into proteoliposomes by SDS-PAGE and immunoblotting with the P-gp antibody C219. a Samples were taken at the indicated time points during the detergent dialysis. Per slot, $0.08 \%$ of the total volume, corresponding to $3.7 \mu \mathrm{g}$ protein of the final preparation $(96 \mathrm{~h})$, was loaded. b The final preparation (after $96 \mathrm{~h}$ of dialysis) was analyzed by sucrose density centrifugation. Of each fraction (1 to 11 , top to bottom) $20 \mu \mathrm{l}$ were loaded on the gel. Fractions 8 and 9 as well as 10 and 11 were pooled.
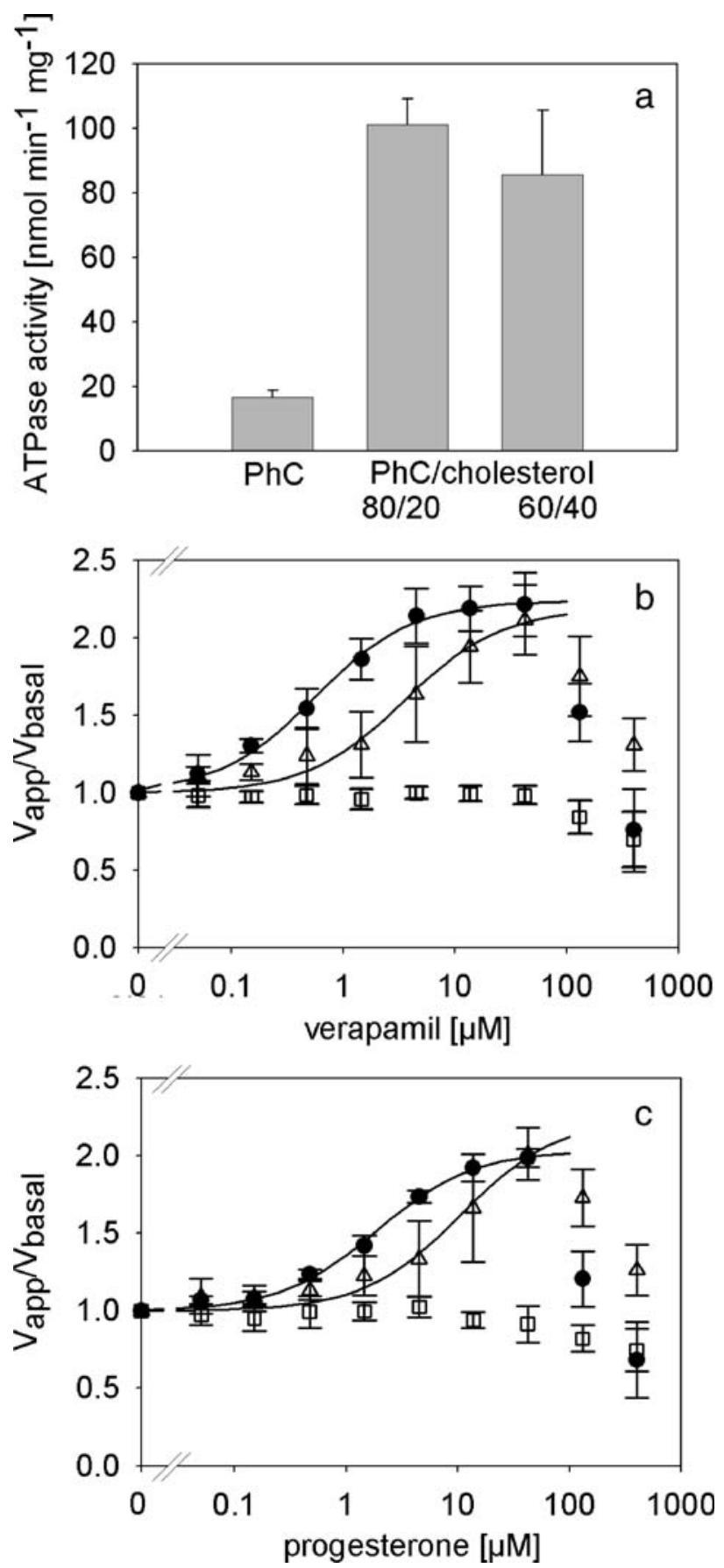

Fig. 2. Influence of cholesterol on the ATPase activity of reconstituted P-gp. The ATPase activities of the P-gp proteoliposomes were determined in the absence (a) and the presence of the modulators verapamil (b) and progesterone (c). (b, c) $\mathrm{PhC}$ proteoliposomes (squares); PhC/cholesterol 80/20 (circles); PhC/cholesterol 60/40 (triangles). The activities shown in $\mathbf{b}$ and $\mathbf{c}$ were normalized to the respective basal activities. The concentration-dependent profiles obtained with the $\mathrm{PhC} /$ cholesterol proteoliposomes were fitted as described in the text (solid lines), the fit parameters are shown in Table II. The symbols are mean values of 6 or 9 activity/ concentration profiles out of 2 or 3 independent experiments. Error bars indicate the standard deviations. 
ATPase activity of P-gp at concentrations $<100 \mu \mathrm{M}$ but have an inhibitory effect at higher concentrations that can be attributed to unspecific effects on the protein or the membrane (12). The ATPase activities of the cholesterolfree and cholesterol-containing proteoliposomes were determined at verapamil and progesterone concentrations between $50 \mathrm{nM}$ and $400 \mu \mathrm{M}$ (Fig. 2b, c). For $\mathrm{PhC}$ proteoliposomes no activation was detected over the whole concentration range while at concentrations $>50 \mu \mathrm{M}$ the ATPase activity was inhibited by both compounds. In the proteoliposomes containing 20 and $40 \%$ cholesterol, respectively, verapamil and progesterone enhanced the ATPase activity at concentrations $<100 \mu \mathrm{M}$. The maximal activities at $50 \mu \mathrm{M}$ modulator were about two-fold higher than the respective basal activities. The activity was decreased at concentrations $>50 \mu \mathrm{M}$. To further analyze the drug induced activity profiles, data were fitted with Eq. 1, excluding the concentrations with the inhibitory effect from the fit. The fits are shown in Fig. 2. The mean ratios of the fitted maximal to basal activities $\left(V^{\prime} / V_{\text {basal }}\right)$ and the mean modulator concentrations at half-maximal activation $(K)$ were derived from the fits of the single experiments and are summarized in Table II. The values for $K$ of verapamil and progesterone were dependent on the cholesterol content of the proteoliposomes while $V^{\prime} / V_{\text {basal }}$ was hardly affected. Both drugs were more potent (had lower $K$ values) in inducing the P-gp ATPase activity in proteoliposomes containing $20 \%$ cholesterol than with $40 \%$ cholesterol. The fitted $K$ of both compounds was about 6 fold lower with $20 \%$ than with $40 \%$ cholesterol.

\section{Influence of the Detergent CHAPS on the ATPase Activity in Egg PhC Proteoliposomes}

Several earlier studies with $\mathrm{P}$-gp reconstituted in $\mathrm{PhC}$ $(7,22,29)$ found a drug-induced increase of the ATPase activity in the absence of cholesterol. Our findings are in contrast to these data as the ATPase activity was not affected by the tested modulators in the absence of cholesterol. The differences between our system and published proteoliposomes are the type and removal method of detergents and the content of residual detergents. We therefore tested the influence of CHAPS, a frequently used detergent for P-gp purification and/or reconstitution, on the ATPase activity of egg $\mathrm{PhC}$ proteoliposomes prepared by our procedure. The final concentration of $28 \mu \mathrm{M}$ CHAPS had no significant influence on the basal ATPase activity, however, with verapamil the typical bell-shaped activity/concentration profile was observed (Fig. 3).

\section{Influence of Cholesterol on the Binding of Verapamil and Progesterone to Reconstituted P-gp}

To investigate whether cholesterol has an effect on the binding of the modulators to P-gp, we studied the affinity of verapamil and progesterone to cholesterol-free and cholesterolcontaining P-gp proteoliposomes by means of equilibrium dialysis at drug concentrations between $10^{-10}$ and $10^{-4} \mathrm{M}$ as described under "Materials and Methods" section. Figure 4 shows the Scatchard plots of both drugs in proteoliposomes containing $20 \%$ cholesterol. The plots revealed two binding classes, one with a high affinity but a low number of interaction sites and a second one with a lower affinity but a higher number of interaction sites. The results were similar for proteoliposomes with $40 \%$ cholesterol. The high affinity was assigned to the binding to $\mathrm{P}$-gp and the low affinity interaction to the partitioning into the lipid bilayer. The binding data were fitted with Eq. 2. The respective fit functions are shown in Fig. 4. The good agreement between the experimental data and the fit function corroborates the underlying model, i.e., the co-existence of a relatively strong specific binding with a low number of binding sites and an unspecific affinity with high capacity. The first is interpreted as the binding to P-glycoprotein, the latter as the membrane affinity. The differences between the fitted parameters of the proteoliposomes with 20 and $40 \%$ cholesterol were within the experimental error of the single experiments. As the purity of the enriched P-gp protein fractions is not known, no absolute binding constants were calculated.

In order to elucidate the reason for the lack of P-gp activation in the cholesterol-free proteoliposomes, we also studied the binding affinity of verapamil and progesterone to $\mathrm{P}$-gp reconstituted in $\mathrm{PhC}$ proteoliposomes. The Scatchard plots shown in Fig. 5 revealed only unspecific binding for both compounds within the tested concentration range (see above). In contrast to the cholesterol-containing proteoliposomes, no increase in binding affinity was detected at the low concentrations. The lack of a distinct slope in the Scatchard plot results from a high number of association sites. The observed affinity was therefore assigned to the partitioning of the drugs into the lipid bilayer. The calculated value of $r / c_{u}$ was in accordance with the partition coefficients revealed with liposomes (see below). In conclusion, no binding of the drugs to P-gp was detectable in the absence of cholesterol.

\section{Influence of Cholesterol on the Lipid Bilayer Affinity of Verapamil and Progesterone}

P-gp is proposed to extract its substrates directly from the inner leaflet of the membrane (2). As the composition of the lipid bilayer has a major influence on the drug-membrane affinity and on the local drug concentration and arrangement inside the membrane, it could affect the drug-P-gp interactions. The influence of cholesterol on the lipid bilayer affinity of verapamil and progesterone was determined with $\mathrm{PhC}$ liposomes containing 0, 20 and 40\% cholesterol, respectively. The membrane affinity of the compounds was measured by means of equilibrium dialysis and verapamil data were fitted using Equation 4. Figure 6 shows the influence of cholesterol on the lipid bilayer partitioning $(D)$ of verapamil (between $\mathrm{pH} 5$ and 12) and progesterone (between $\mathrm{pH} 2$ and 12), respectively, and on the fitted partition coefficients of the neutral compounds $\left(P^{N}\right)$ and of protonated verapamil $\left(P^{I}\right)$. The $P^{N}$ of progesterone was decreased 1.8 fold upon incorporation of $20 \%$ cholesterol in $\mathrm{PhC}$ liposomes. Increasing the cholesterol content to $40 \%$ led to a further decrease of $P^{N}$ by a factor of 1.6. In the case of verapamil, the presence of $20 \%$ cholesterol decreased the affinity of the neutral species $\left(P^{N}\right) 1.2$ fold, increasing the cholesterol to $40 \%$ led to a further decrease by a factor of 2.4. The affinity of the protonated verapamil $\left(P^{I}\right)$ was decreased 1.8 fold by incorporation of $20 \%$ cholesterol and 1.4 fold by increasing the amount of cholesterol from 20 to $40 \%$. 
Table II. Influence of Cholesterol on the Basal and Drug-induced P-gp ATPase Activity

\begin{tabular}{|c|c|c|c|c|c|c|c|}
\hline & \multirow{2}{*}{$V_{\text {basal }}^{a}$} & \multicolumn{2}{|l|}{$V^{\prime b}$} & \multicolumn{2}{|l|}{$V^{\prime} / V_{\text {basal }}^{b}$} & \multicolumn{2}{|l|}{$K^{b}$} \\
\hline & & Verap & Progest & Verap & Progest & Verap & Progest \\
\hline $\mathrm{PhC}$ & $16.6 \pm 2.1$ & - & - & - & - & - & - \\
\hline PhC:Chol 80:20 & $101.0 \pm 8.1$ & $227.0 \pm 14.8$ & $196.7 \pm 15.9$ & $2.24 \pm 0.02$ & $2.04 \pm 0.02$ & $0.56 \pm 0.05$ & $1.88 \pm 0.12$ \\
\hline PhC:Chol 60:40 & $85.6 \pm 20.0$ & $182.1 \pm 42.7$ & $175.8 \pm 44.1$ & $2.19 \pm 0.05$ & $2.24 \pm 0.08$ & $3.56 \pm 0.49$ & $10.58 \pm 1.81$ \\
\hline
\end{tabular}

$V_{\text {basal }}$ and $V^{\prime}\left[\mathrm{nmol} \times \min ^{-1} \times \mathrm{mg}^{-1}\right] ; K[\mu \mathrm{M}]$

Verap, verapamil; Progest, progesterone

${ }^{a}$ Mean values \pm standard deviations $(n \geq 36) ;{ }^{b}$ means of fitted values \pm standard deviations from six or nine activity/concentration profiles out of two or three independent experiments each.

\section{DISCUSSION}

The present study was conducted with P-gp proteoliposomes which contained negligible amounts of residual detergents. As P-gp was solubilized and purified with SDS according to Dong et al. (33) it can also be expected that no residual lipids from the $\mathrm{P}$-gp source were present. The absence of significant amounts of detergents and source lipids makes this system unique and allows to investigate the influence of the lipid environment and of drug compounds on $\mathrm{P}$-gp functions under well defined conditions.

Residual detergents in P-gp proteoliposomes may significantly influence the ATPase activity of the transporter (31). The detergent CHAPS is frequently used for the purification and reconstitution of P-gp. Romsicki and Sharom (42) studied the influence of additional CHAPS on the ATPase activity of $\mathrm{P}$-gp in di-myristoyl $\mathrm{PhC}$ (DMPC) proteoliposomes prepared from P-gp/lipid/CHAPS micelles. The residual detergent concentration was $<0.02 \%$ of the starting concentration, corresponding to a concentration of $<40 \mu \mathrm{M}$. The addition of more detergent $(\geq \sim 30 \mu \mathrm{M})$ led to a significant increase in the ATPase activity. The authors assigned this effect to the permeabilization of the vesicles. However, these data could also disclose the influence of the

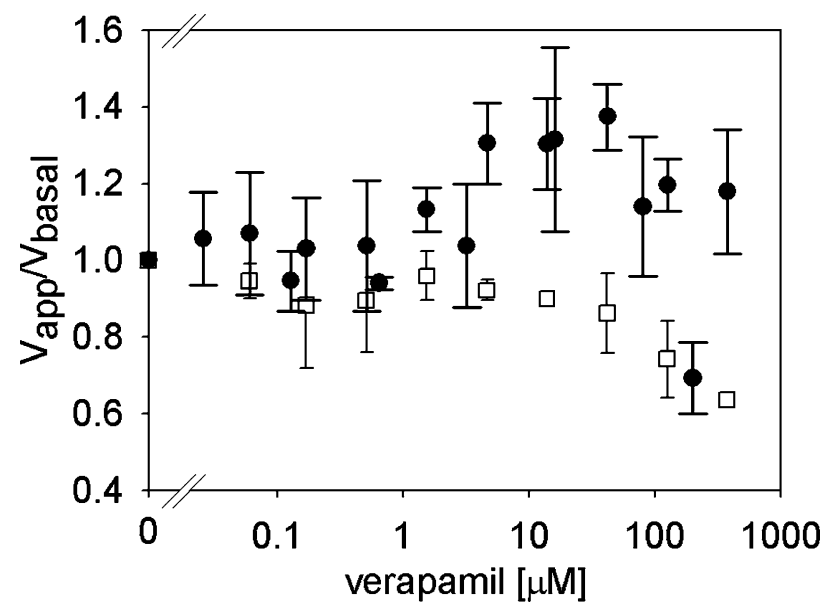

Fig. 3. Influence of CHAPS on the ATPase activity of P-gp in egg $\mathrm{PhC}$ proteoliposomes at different verapamil concentrations. Circles, after incubation of the proteoliposomes with CHAPS, the final detergent concentration in the assay was $28 \mu \mathrm{M}$. Squares, influence of verapamil in the absence of detergent. Shown are mean values \pm standard deviations of 4 independent experiments each. The basal activity after CHAPS incubation did not significantly differ from the activity in the absence of CHAPS. detergent on the ATPase activity of the protein. CHAPS restores P-gp ATPase activity even in the absence of lipids (31) and verapamil was able to increase the ATPase activity of P-gp in $0.4 \mathrm{mM}$ of the detergent (32).

In our egg $\mathrm{PhC}$ proteoliposomes the P-gp ATPase activity was not inducible by verapamil and progesterone in the absence of cholesterol. This is in contrast to the findings of other studies with PhC proteoliposomes $(7,22,29)$. However, the incubation with CHAPS restored the verapamil-
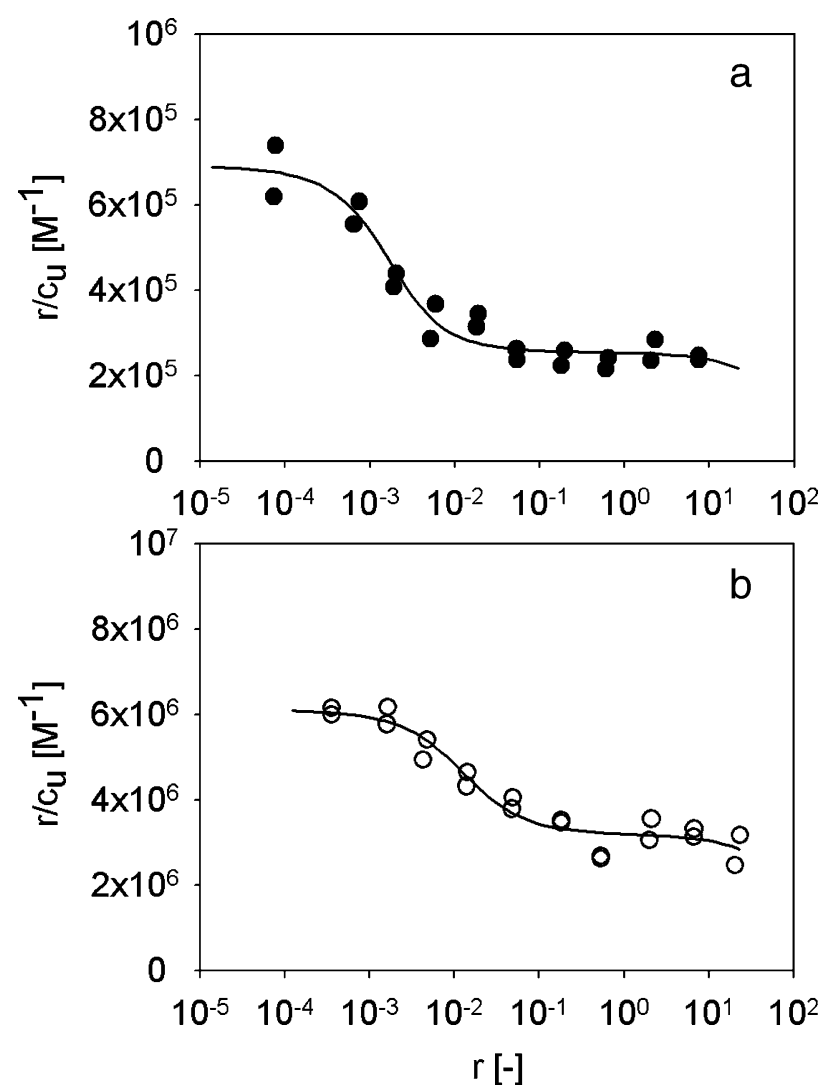

Fig. 4. Scatchard plots of verapamil and progesterone binding to P-gp $\mathrm{PhC/cholesterol} \mathrm{80/20} \mathrm{proteoliposomes.} \mathrm{Verapamil} \mathrm{(a)} \mathrm{and} \mathrm{progester-}$ one (b) were incubated at various concentrations between $10^{-10}$ and $10^{-4} \mathrm{M}$ with proteoliposomes (protein concentration between 0.1 and $0.5 \mathrm{mg} / \mathrm{ml}$ ). Binding was determined by equilibrium dialysis at $37^{\circ} \mathrm{C}$. Solid lines show the fit functions for two binding classes as described in the text. Results are from one representative experiment each. Proteoliposomes with $40 \%$ cholesterol revealed similar Scatchard plots. 


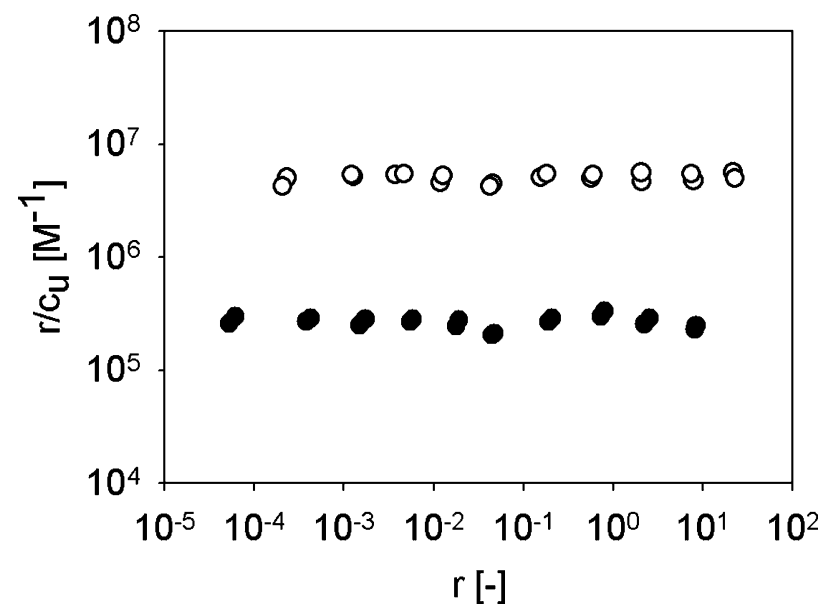

Fig. 5. Scatchard plots for the binding of verapamil and progesterone to P-gp proteoliposomes in the absence of cholesterol. Verapamil (closed circles) and progesterone (open circles) binding to P-gp PhC proteoliposomes was determined as described in Fig. 4 and in the text. Only unspecific binding, i.e., partitioning into the lipid bilayer, was detected as seen from the concentration-independent $r / c_{u}$ values. Data are from one representative experiment each.

inducible ATPase activity in the absence of cholesterol in our assay. This indicates that P-gp is potentially functional in egg $\mathrm{PhC}$ proteoliposomes. It confirms that the non-inducibility by verapamil and progesterone, respectively, is indeed due to the absence of cholesterol or any other membrane constituent which induces functionality of the ATPase and not due to irreversible inactivation during the purification and reconstitution procedures. It further emphasizes the influence of residual detergent on the ATPase activity of the transporter. The overlapping effect of cholesterol and CHAPS on the function of P-gp, as found in our study, is not surprising, considering the similarity in their molecular structure.

We can not exclude that P-gp is partially denatured in our system due to the contact with the relatively strong detergents SDS and cholate. However, the increase in ATPase activity during dialysis and the typical behavior of the $\mathrm{PhC} /$ cholesterol proteoliposomes in terms of ATPase activity and activation by the modulators, indicates that at least a significant part of the transporter is correctly folded. Investigations by Dong et al. (33) and by Naito and Tsuruo (57) have shown before that P-gp which was solubilized with SDS or cholate regains its function after the removal of the detergents.

The tremendous increase in the basal ATPase activity caused by cholesterol is in line with all discussed possible functions of the sterol: (a) It could be transported by P-gp and therefore increase its ATPase activity. (b) Cholesterol could affect the conformation and/or conformational flexibility of P-gp, i.e., freedom to change conformation upon drug or ATP binding, by a direct interaction and therefore enable its functionality as an ATPase without being transported. (c) Cholesterol could indirectly affect the conformation and conformational flexibility of P-gp via its influence on the membrane properties, and therefore induce its activity as an ATPase.

The binding to P-gp and the enhancement of the P-gp ATPase activity by the two modulators verapamil and progesterone was only observed in the presence of choles- terol in the bilayer. This indicates that cholesterol has a direct or indirect effect on the protein conformation, enabling the interaction with the modulators. This effect would be independent of the recognition of cholesterol as a transport substrate by P-gp but would correspond to the above discussed possible functions (b) and (c), respectively, of cholesterol.

Saeki et al. (29) found a similar influence of cholesterol on azidopine binding to a reconstituted P-gp fusion protein. Evidence that the membrane environment affects the efficiency of allosteric conformational changes of the protein was demonstrated by Modok et al. (22). Their data suggest that a raft-like lipid composition enhances the drug transporter activity of P-gp.

Direct binding of cholesterol to P-gp could lead to the above discussed conformational change in the protein (b). A direct interaction of cholesterol with the P-gp site of daunorubicin binding was suggested by Wang et al. (19) based on ATPase assays with membrane vesicles from P-gp overexpressing cells. The authors found a mixed type (noncompetitive) inhibition of the daunorubicin-induced ATPase activity by cholesterol.
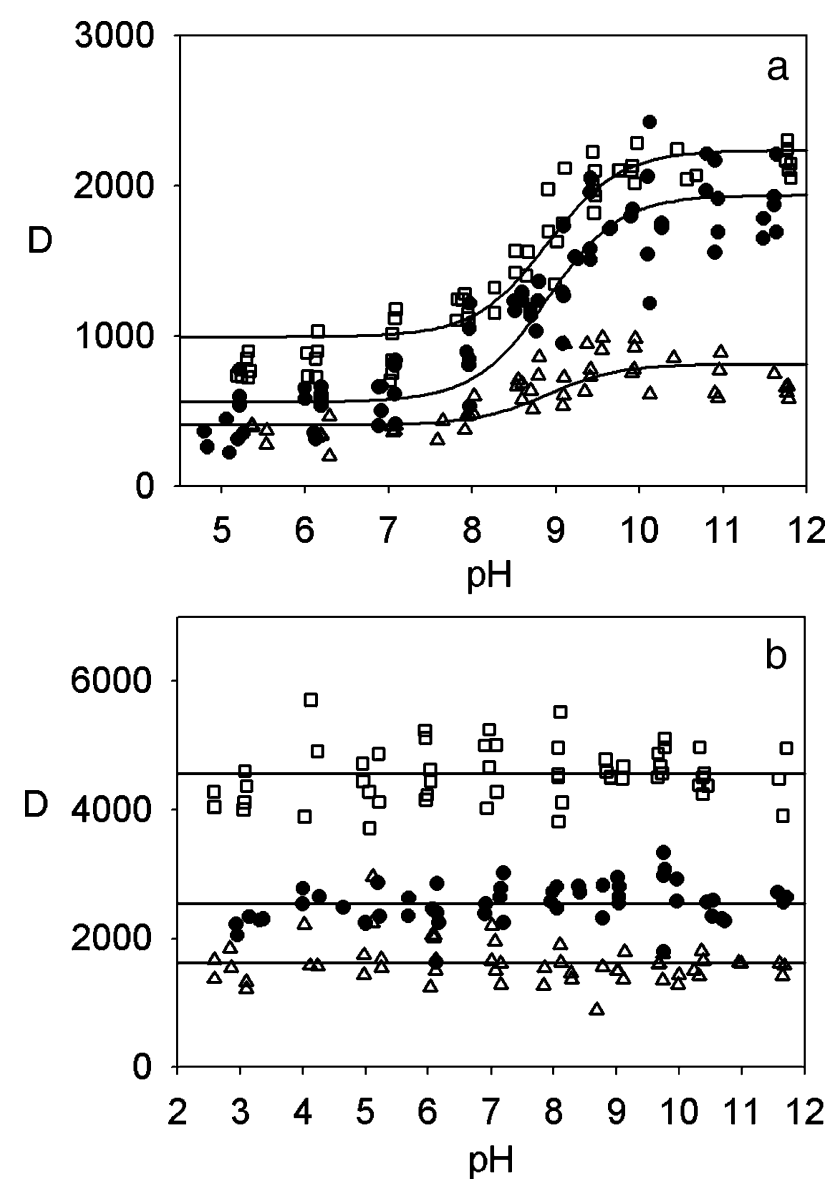

Fig. 6. Influence of cholesterol on the lipid bilayer affinities of verapamil and progesterone. The $\mathrm{pH}$-dependent partitioning of verapamil (a) and progesterone (b) was determined with $\mathrm{PhC}$ (squares), PhC/cholesterol 80/20 (circles) and $\mathrm{PhC} /$ cholesterol 60/40 (triangles) liposomes. The solid lines show the fit functions as described in the text for (a) and mean values for (b). 
Further evidence of a direct interaction between cholesterol and P-gp was described by Kimura et al. (14). Cholesterol bound to P-gp in the absence of lipids. This could, however, be different within the lipid bilayer, considering the high affinity of the sterol to phospholipids and the interaction between P-gp and phospholipids. Both equilibria are in competition with the binding between cholesterol and $\mathrm{P}$-gp. It is still open how cholesterol distributes between P-gp and the lipid environment.

An indirect effect of cholesterol on the conformation of P-gp (c) could arise from its influence on the bilayer rigidity and structure (21) or on the membrane thickness. The addition of cholesterol to egg $\mathrm{PhC}$ bilayers could lead to a favorable hydrophobic matching of the bilayer and the protein, enabling its optimal function as an ATPase. Inclusion of cholesterol to egg $\mathrm{PhC}$ bilayers increases the hydrophobic thickness of the membrane from 26 to $33 \AA$ $(43,44)$ which better matches the hydrophobic core of P-gp that has been estimated to be $34 \AA$ (45). A hydrophobic mismatch could lead to a change in the transmembrane orientation of the $\alpha$-helices, distribution of conformational states or conformational mobility of the transmembrane domains (46). This could affect P-gp activity as the protein undergoes large conformational rearrangements during its transport cycle (47).

Further insight into the effect of cholesterol on P-gp function is given by the comparison of the kinetics in the presence of 20 and $40 \%$ cholesterol in the bilayers. In our study, the modulator concentrations at half maximal induction of the ATPase activity $(K)$ are dependent on the membrane content of cholesterol. They are higher with $40 \%$ cholesterol than with $20 \%$. The membrane affinities of both modulators are higher with $20 \%$ cholesterol than with $40 \%$. Assuming Michaelis-Menten conditions, $K$ is related to the binding affinity of the modulators to P-gp. In this case, cholesterol would influence the binding of the modulators to P-gp. Alternatively, the differences in $K$ could reflect differences in processes such as the release of the modulator after ATP hydrolysis or the interaction of the protein with ATP. In addition, $K$ could be affected by the membrane affinity, if one (or both) of the partitioning rate constants is ratedetermining for the binding to the active protein or the release from the binding site. However, increasing the cholesterol content from 20 to $40 \%$ had a higher impact on the concentrations $K$ than on the membrane affinities $D$ of the two modulators. This makes it unlikely that the partitioning kinetics are rate-limiting for the P-gp binding kinetics. We would also like to note here, that the membrane affinity must not necessarily be positively related with the P-gp binding affinity. An increase in membrane affinity caused by the lipid composition is at the cost of both equilibria the drug partitioning between the water phase and the membrane as well as the drug partitioning between the protein and the membrane. At equilibrium, the protein binding constant (determined from the protein bound concentration and the aqueous concentration) may be independent of the membrane affinity.

The maximal ATPase activities $\left(V^{\prime}\right)$ induced by verapamil and progesterone in the presence of 20 and $40 \%$ cholesterol, respectively, were all similar, indicating that the $\mathrm{P}$-gp function and therefore conformation is unchanged comparing the two membrane environments. The fact that the cholesterol content $(\geq 20 \%)$ has an influence on $K$ but has no influence on the functionality of P-gp, indicates that cholesterol-in addition to enhancing the basal ATPase activity and rendering the protein sensitive towards the modulators-may indeed interact with the binding sites of the modulators in a competitive manner.

Kimura et al. (14) observed a similar influence of cholesterol on $K$ and $V$ ' of verapamil with reconstituted P-gp. Whether $K$ was affected by cholesterol was dependent on the molecular weight of the tested compounds in their study. While cholesterol affected the $K$ of verapamil (mw 455) it had no effect on the $K$ of paclitaxel (mw 854). Their model suggests that cholesterol may fill up the binding site when small compounds, such as verapamil bind to P-gp, but is excluded from the binding site when larger compounds, such as paclitaxel bind. In their system P-gp was activated by the tested compounds in the absence of cholesterol while P-gp was not activated under these conditions in our study. It will be interesting to see whether paclitaxel and other larger compounds can activate $\mathrm{P}$-gp under our conditions in the absence of cholesterol. This would support the model of Kimura et al., i.e., that cholesterol is only involved in the binding and activation by small compounds, such as verapamil.

Comparing the concentrations of cholesterol and modulator within the bilayer at maximal ATPase activation in our study, the model of competitive binding appears realistic. The local modulator concentrations within the membrane at maximal ATPase activation are about $50 \mathrm{mM}$ as estimated from the liposome partition coefficients $D\left(\sim 10^{3}\right)$ and the aqueous modulator concentrations $(50 \mu \mathrm{M})$. The local cholesterol concentration within the bilayer is in the range of $260-500 \mathrm{mM}$ (the total lipid concentration within the bilayer is $\sim 1.3 \mathrm{M}$ ). Depending on the protein binding affinity of the modulator and of cholesterol, respectively, and on their respective affinities to the membrane lipids-which may compete with the protein binding-a significant competition between cholesterol and the modulator for a P-gp binding site is possible. This model of competitive binding is in agreement with the increased $K$ at the higher cholesterol/ lipid ratio.

Romsicki and Sharom (7) found a good correlation between P-gp binding affinities and membrane affinities with vinblastine, verapamil and daunorubicin in egg $\mathrm{PhC}$, DMPC and di-palmitoyl PhC (DPPC) membranes (note that these proteoliposomes do not contain cholesterol but may contain residual CHAPS). In the same work the membrane affinity and P-gp binding affinity, respectively, of verapamil did not appear to affect $K$ in the respective proteoliposomes. In turn, the ratio between the maximal inducible ATPase activity and the basal activity $\left(V^{\prime} / V_{\text {basal }}\right)$ was lipid-dependent. These findings could point to an effect of the lipid environment on the protein which is not directly related to the drug binding site, i.e., an effect of incompetitive character. In their study, the lipid environment could affect the P-gp function in dependence of the lipid chain length and order, which determine the thickness and rigidity of the bilayer.

In general, further effects of cholesterol may occur and be relevant for $\mathrm{P}$-gp function: cholesterol may be needed as a substrate for counter transport when drug substrates or modulators are moved from the cytoplasmic to the exoplas- 
mic membrane leaflet by P-gp. Cholesterol and the lipid composition in general, could bring $\mathrm{P}$-gp substrates or modulators in a position within the bilayer which is favorable for their recognition by P-gp. An inhomogeneous distribution of the lipids within the bilayer, e.g., an increase of cholesterol in the vicinity of P-gp, could affect the local distribution of drug compound within the membrane. Finally, P-gp is discussed as a phopholipid transporter $(23,48)$ and could therefore have an indirect effect on the distribution of cholesterol between the two membrane leaflets.

Based on our results and on studies cited in this work, one could speculate that cholesterol is transported by $\mathrm{P}$-gp and regulates the P-gp transport (and ATPase) function via its content within the bilayer. In the absence of cholesterol, P-gp displays low activity and only in the presence of cholesterol, P-gp gets fully functional and regulates the distribution of the sterol between the cytosolic and exosolic membrane leaflets. In this conformation, P-gp is further activated by the modulators verapamil and progesterone, which compete with cholesterol for the binding to the transporter. Further investigations have to show whether other membrane constituents than cholesterol can activate P-gp and whether other compounds than those we tested can interact with P-gp in the absence of the sterol.

Considering the substantial influence of the membrane environment on P-gp, findings with reconstituted P-gp have to be interpreted in the light of all system components. Detergents and additional adjuvants from the purification and reconstitution procedures can affect the behavior of the transporter, as we have shown for CHAPS. The most frequently used detergents for the purification of P-gp beside CHAPS $(26,28,29,32,39,42)$ are n-octyl $\beta$-D-glucopyranoside (octyl glucoside) $(27,30,49-53)$ and n-dodecyl or n-decyl $\beta$-Dmaltoside $(14,22,25,33,51,54-56)$. Octyl glucoside had activating as well as inhibiting effects on the ATPase activity of CHAPS-solubilized P-gp, depending on the glucoside concentration (39). Alternatively, other detergents such as cholates (57), lyso $\mathrm{PhC}$ (58) and some non-ionic detergents (59) were investigated. The influence of most of these detergents on membrane-reconstituted P-gp remains to be investigated. Several systems contain protease inhibitors in addition and preparations of histidine-tagged P-gp generally contain considerable amounts of imidazole. These additional compounds are potential substrates, modulators or inhibitors of P-gp. Their assay concentrations are generally not known and their influence on the P-gp behavior is rarely examined.

The lipid composition of the reconstituted system is crucial for the behavior of the transporter. Depending on the purification technique the protein is delipidated to different degrees. Lipid compositions for reconstitution range from single phospholipids of synthetic or natural origin to lipid mixtures of phospholipids with or without cholesterol.

Beside reconstituted P-gp, membrane vesicles of P-gpexpressing cells are used to investigate $\mathrm{P}$-gp characteristics. Depending on the cell type, the lipid composition can vary strikingly. Membrane vesicles were prepared from mammalian cells (60-64) and from P-gp-transfected insect cells $(65,66)$. In the light of our findings it is interesting to note that plasma membranes from insect cells (Sf9) contain about ten times less cholesterol than plasma membranes from higher eukaryotic cells (67).
In conclusion, cholesterol exerts three effects on P-gp: (a) it increases its basal ATPase activity, (b) it enables P-gp binding and activation by the modulators verapamil and progesterone and (c) it may compete with verapamil and progesterone for the same binding site(s).

\section{REFERENCES}

1. S. Orlowski, S. Martin, and A. Escargueil. P-glycoprotein and 'lipid rafts': some ambiguous mutual relationships (floating on them, building them or meeting them by chance?). Cell. Mol. Life Sci. 63:1038-1059 (2006).

2. S. V. Ambudkar, I. W. Kim, and Z. E. Sauna. The power of the pump: mechanisms of action of P-glycoprotein (ABCB1). Eur. J. Pharm. Sci. 27:392-400 (2006).

3. M. F. Fromm. Importance of P-glycoprotein at blood-tissue barriers. Trends Pharmacol. Sci. 25:423-429 (2004).

4. S. Vandevuer, F. Van Bambeke, P. M. Tulkens, and M. Prevost. Predicting the three-dimensional structure of human P-glycoprotein in absence of ATP by computational techniques embodying crosslinking data: insight into the mechanism of ligand migration and binding sites. Proteins 63:466-478 (2006).

5. A. Rothnie, J. Storm, R. McMahon, A. Taylor, I. D. Kerr, and R. Callaghan. The coupling mechanism of P-glycoprotein involves residue L339 in the sixth membrane spanning segment. FEBS Lett. 579:3984-3990 (2005).

6. P. Hafkemeyer, S. Dey, S. V. Ambudkar, C. A. Hrycyna, I. Pastan, and M. M. Gottesman. Contribution to substrate specificity and transport of nonconserved residues in transmembrane domain 12 of human P-glycoprotein. Biochemistry 37:16400-16409 (1998).

7. Y. Romsicki and F. J. Sharom. The membrane lipid environment modulates drug interactions with the P-glycoprotein multidrug transporter. Biochemistry 38:6887-6896 (1999).

8. T. W. Loo, M. C. Bartlett, and D. M. Clarke. ATP hydrolysis promotes interactions between the extracellular ends of transmembrane segments 1 and 11 of human multidrug resistance $\mathrm{P}$ glycoprotein. Biochemistry 44:10250-10258 (2005).

9. J. Ferté. Analysis of the tangled relationships between Pglycoprotein-mediated multidrug resistance and the lipid phase of the cell membrane. Eur. J. Biochem. 267:277-294 (2000).

10. G. D. Eytan. Mechanism of multidrug resistance in relation to passive membrane permeation. Biomed. Pharmacother. 59:90-97 (2005).

11. S. W. Kamau, S. D. Krämer, M. Günthert, and H. WunderliAllenspach. Effect of the modulation of the membrane lipid composition on the localization and function of P-glycoprotein in MDR1-MDCK cells. In Vitro Cell. Dev. Biol. 41:207-216 (2005).

12. K. Bucher, C. A. Besse, S. W. Kamau, H. Wunderli-Allenspach, and S. D. Krämer. Isolated rafts from adriamycin-resistant P388 cells contain functional ATPases and provide an easy test system for P-glycoprotein-related activities. Pharm. Res. 22:449-457 (2005).

13. G. D. Luker, C. M. Pica, S. Kumar, D. F. Covey, and D. PiwnicaWorms. Effects of cholesterol and enantiomeric cholesterol on P-glycoprotein localization and function in low-density membrane domains. Biochemistry 39:7645-7846 (2000).

14. Y. Kimura, N. Kioka, H. Kato, M. Matsuo, and K. Ueda. Modulation of drug-stimulated ATPase activity of human MDR1/ P-glycoprotein by cholesterol. Biochem. J. 401:597-605 (2007).

15. S. Meyer Dos Santos, C.-C. Weber, C. Franke, W. E. Müller, and G. P. Eckert. Cholesterol: coupling between membrane microenvironment and ABC transporter activity. Biochem. Biophys. Res. Commun. 354:216-221 (2007).

16. L. Gayet, G. Dayan, S. Barakat, S. Labialle, M. Michaud, S. Cogne, A. Mazane, A. W. Coleman, D. Rigal, and L. G. Baggetto. Control of P-glycoprotein activity by membrane cholesterol amounts and their relation to multidrug resistance in human CEM leukemia cells. Biochemistry 44:4499-4509 (2005). 
17. J. Troost, H. Lindenmaier, W. E. Haefeli, and J. Weiss. Modulation of cellular cholesterol alters P-glycoprotein activity in multidrug-resistant cells. Mol. Pharmacol. 66:1332-1339 (2004).

18. A. Garrigues, A. E. Escargueil, and S. Orlowski. The multidrug transporter, P-glycoprotein, actively mediates cholesterol redistribution in the cell membrane. Proc. Natl. Acad. Sci. U. S. A. 99:10347-10352 (2002).

19. E. J. Wang, C. N. Casciano, R. P. Clement, and W. W. Johnson. Cholesterol interaction with the daunorubicin binding site of $\mathrm{P}$ glycoprotein. Biochem. Biophys. Res. Commun. 276:909-916 (2000).

20. T. G. Tessner and W. F. Stenson. Overexpression of MDR1 in an intestinal cell line results in increased cholesterol uptake from micelles. Biochem. Biophys. Res. Commun. 267:565-571 (2000).

21. O. G. Mouritsen and M. J. Zuckermann. What's so special about cholesterol?. Lipids 39:1101-1113 (2004).

22. S. Modok, C. Heyward, and R. Callaghan. P-glycoprotein retains function when reconstituted into a sphingolipid- and cholesterolrich environment. J. Lipid Res. 45:1910-1918 (2004).

23. Y. Romsicki and F. J. Sharom. Phospholipid flippase activity of the reconstituted P-glycoprotein multidrug transporter. Biochemistry 40:6937-6947 (2001).

24. Y. Romsicki and F. J. Sharom. The ATPase and ATP-binding functions of P-glycoprotein-Modulation by interaction with defined phospholipids. Eur. J. Biochem. 256:170-178 (1998).

25. A. Rothnie, D. Theron, L. Soceneantu, C. Martin, M. Traikia, G. Berridge, C. F. Higgins, P. F. Devaux, and R. Callaghan. The importance of cholesterol in maintenance of P-glycoprotein activity and its membrane perturbing influence. Eur. Biophys. J. 30:430-442 (2001).

26. P. Lu, R. Liu, and F. J. Sharom. Drug transport by reconstituted P-glycoprotein in proteoliposomes. Effect of substrates and modulators, and dependence on bilayer phase state. Eur. J. Biochem. 268:1687-1697 (2001).

27. I. L. Urbatsch and A. E. Senior. Effects of lipids on Atpase activity of purified Chinese-Hamster P-glycoprotein. Arch. Biochem. Biophys. 316:135-140 (1995).

28. F. J. Sharom, X. H. Yu, and C. A. Doige. Functional reconstitution of drug transport and ATPase activity in proteoliposomes containing partially purified P-glycoprotein. J. Biol. Chem. 268:24197-24202 (1993).

29. T. Saeki, A. M. Shimabuku, K. Ueda, and T. Komano. Specific drug-binding by purified lipid-reconstituted P-glycoproteinDependence on the lipid-composition. Biochim. Biophys. Acta. 1107:105-110 (1992)

30. S. Ambudkar, I. Lelong, J. Zhang, C. Cardarelli, M. Gottesman, and I. Pastan. Partial purification and reconstitution of the human multidrug-resistance pump: characterization of the drugstimulatable ATP hydrolysis. Proc. Natl. Acad. Sci. U. S. A. 89:8472-8476 (1992).

31. S. Orlowski, M. A. Selosse, C. Boudon, C. Micoud, L. M. Mir, J. Belehradek Jr., and M. Garrigos. Effects of detergents on Pglycoprotein ATPase activity: differences in perturbations of basal and verapamil-dependent activities. Cancer Biochem. Biophys. 16:85-110 (1998).

32. F. J. Sharom, X. H. Yu, J. W. K. Chu, and C. A. Doige. Characterization of the ATPase activity of P-glycoprotein from multidrug-resistant chinese-hamster ovary cells. Biochem. J. 308:381-390 (1995).

33. M. Q. Dong, F. Penin, and L. G. Baggetto. Efficient purification and reconstitution of P-glycoprotein for functional and structural studies. J. Biol. Chem. 271:28875-28883 (1996).

34. S. D. Krämer, A. Braun, C. Jakits-Deiser, and H. WunderliAllenspach. Towards the predictability of drug-lipid membrane interactions: the $\mathrm{pH}$-dependent affinity of propranolol to phosphatidylinositol containing liposomes. Pharm. Res. 15:739-744 (1998).

35. O. Zumbühl and H. G. Weder. Liposomes of controllable size in the range of 40 to $180 \mathrm{~nm}$ by defined dialysis of lipid/detergent mixed micelles. Biochim. Biophys. Acta. 640:252-262 (1981).

36. R. Singh, M. Ajagbe, S. Bhamidipati, Z. Ahmad, and M. Ahmad. A rapid isocratic high-performance liquid chromatography method for determination of cholesterol and 1,2-dioleoylsn-glycero-3-phosphocholine in liposome-based drug formulations. J. Chromatogr. 1073:347-353 (2005).

37. Q. Qu, J. W. K. Chu, and F. J. Sharom. Transition state Pglycoprotein binds drugs and modulators with unchanged affinity, suggesting a concerted transport mechanism. Biochemistry 42:1345-1353 (2003).

38. G. M. Pauletti and H. Wunderli-Allenspach. Partition coefficients in vitro: artificial membranes as a standardized distribution model. Eur. J. Pharm. Sci. 1:273-282 (1994).

39. C. A. Doige, X. Yu, and F. J. Sharom. The effects of lipids and detergents on ATPase-active P-glycoprotein. Biochim. Biophys. Acta. 1146:65-72 (1993).

40. J. M. H. van den Elsen, D. A. Kuntz, F. J. Hoedemaeker, and D. R Rose. Antibody C219 recognizes an alpha-helical epitope on Pglycoprotein. Proc. Natl. Acad. Sci. U. S. A. 96:13679-13684 (1999).

41. B. Mukherjee, B. Patra, S. Mahapatra, P. Banerjee, A. Tiwari, and M. Chatterjee. Vanadium - an element of atypical biological significance. Toxicol. Lett. 150:135-143 (2004).

42. Y. Romsicki and F. J. Sharom. Interaction of P-glycoprotein with defined phospholipid bilayers: a differential scanning calorimetric study. Biochemistry 36:9807-9815 (1997).

43. V. A. Parsegian, N. Fuller, and R. P. Rand. Measured work of deformation and repulsion of lecithin bilayers. Proc. Natl. Acad. Sci. U. S. A. 76:2750-2754 (1979).

44. L. J. Lis, M. Mcalister, N. Fuller, R. P. Rand, and V. A. Parsegian. Interactions between neutral phospholipid-bilayer membranes. Biophys. J. 37:657-665 (1982).

45. M. F. Rosenberg, G. Velarde, R. C. Ford, C. Martin, G Berridge, I. D. Kerr, R. Callaghan, A. Schmidlin, C. Wooding, K. J. Linton, and C. F. Higgins. Repacking of the transmembrane domains of P-glycoprotein during the transport ATPase cycle. EMBO J. 20:5615-5625 (2001).

46. J. H. Ren, S. Lew, J. Y. Wang, and E. London. Control of the transmembrane orientation and interhelical interactions within membranes by hydrophobic helix length. Biochemistry 38:59055912 (1999).

47. A. Rothnie, J. Storm, J. Campbell, K. J. Linton, I. D. Kerr, and R. Callaghan. The topography of transmembrane segment six is altered during the catalytic cycle of P-glycoprotein. J. Biol. Chem. 279:34913-34921 (2004).

48. A. van Helvoort, M. L. Giudici, M. Thielemans, and G. van Meer. Transport of sphingomyelin to the cell surface is inhibited by brefeldin A and in mitosis, where C-6-NBD-sphingomyelin is translocated across the plasma membrane by a multidrug transporter activity. J. Cell Sci. 110:75-83 (1997).

49. G. D. Eytan, M. J. Borgnia, R. Regev, and Y. G. Assaraf Transport of polypeptide ionophores into proteoliposomes reconstituted with rat-liver P-glycoprotein. J. Biol. Chem. 269:26058-26065 (1994).

50. A. B. Shapiro and V. Ling. ATPase activity of purified and reconstituted P-glycoprotein from chinese-hamster ovary cells. J. Biol. Chem. 269:3745-3754 (1994).

51. M. F. Rosenberg, R. Callaghan, R. C. Ford, and C. F. Higgins. Structure of the multidrug resistance P-glycoprotein to $2.5 \mathrm{~nm}$ resolution determined by electron microscopy and image analysis. J. Biol. Chem. 272:10685-10694 (1997).

52. M. Ramachandra, S. V. Ambudkar, D. Chen, C. A. Hrycyna, S. Dey, M. M. Gottesman, and I. Pastan. Human P-glycoprotein exhibits reduced affinity for substrates during a catalytic transition state. Biochemistry 37:5010-5019 (1998).

53. Z. E. Sauna, K. Nandigama, and S. V. Ambudkar. Exploiting reaction intermediates of the ATPase reaction to elucidate the mechanism of transport by P-glycoprotein (ABCB1). J. Biol. Chem. 281:26501-26511 (2006).

54. T. W. Loo and D. M. Clarke. The minimum functional unit of human P-glycoprotein appears to be a monomer. J. Biol. Chem. 271:27488-27492 (1996).

55. M. Julien and P. Gros. Nucleotide-induced conformational changes in P-glycoprotein and in nucleotide binding site mutants monitored by trypsin sensitivity. Biochemistry 39:4559-4568 (2000).

56. E. M. Howard and P. D. Roepe. Purified human MDR 1 modulates membrane potential in reconstituted proteoliposomes. Biochemistry 42:3544-3555 (2003).

57. M. Naito and T. Tsuruo. Reconstitution of purified P-glycoprotein into liposomes. J. Cancer Res. Clin. 121:582-586 (1995).

58. Q. C. Mao and G. A. Scarborough. Purification of functional human P-glycoprotein expressed in Saccharomyces cerevisiae. Biochim. Biophys. Acta 1327:107-118 (1997). 
59. R. Callaghan, G. Berridge, D. R. Ferry, and C. F. Higgins. The functional purification of $\mathrm{P}$-glycoprotein is dependent on maintenance of a lipid-protein interface. Biochim. Biophys. Acta 1328:109-124 (1997).

60. M. K. Al-shawi and A. E. Senior. Characterization of the adenosine-triphosphatase activity of chinese-hamster P-glycoprotein. J. Biol. Chem. 268:4197-4206 (1993).

61. S. Orlowski, L. M. Mir, J. Belehradek, and M. Garrigos. Effects of steroids and verapamil on P-glycoprotein ATPase activity: progesterone, desoxycorticosterone, corticosterone and verapamil are mutually non-exclusive modulators. Biochem. J. 317:515522 (1996).

62. M. Zhang, G. C. Wang, A. Shapiro, and J. T. Zhang. Topological folding and proteolysis profile of P-glycoprotein in membranes of multidrug-resistant cells: implications for the drug-transport mechanism. Biochemistry 35:9728-9736 (1996).

63. R. L. Shepard, M. A. Winter, S. C. Hsaio, H. L. Pearce, W. T. Beck, and A. H. Dantzig. Effect of modulators on the ATPase activity and vanadate nucleotide trapping of human P-glycoprotein. Biochem. Pharmacol. 56:719-727 (1998).

64. M. Bebawy, M. B. Morris, and B. D. Roufogalis. A continuous fluorescence assay for the study of P-glycoprotein-mediated drug efflux using inside-out membrane vesicles. Anal. Biochem. 268:270-277 (1999).

65. M. Müller, E. Bakos, E. Welker, A. Varadi, U. A. Germann, M. M. Gottesman, B. S. Morse, I. B. Roninson, and B. Sarkadi. Altered drug-stimulated ATPase activity in mutants of the human multidrug resistance protein. J. Biol. Chem. 271:1877-1883 (1996).

66. K. Szabo, E. Bakos, E. Welker, M. Muller, H. R. Goodfellow, C. F. Higgins, A. Varadi, and B. Sarkadi. Phosphorylation site mutations in the human multidrug transporter modulate its drug-stimulated ATPase activity. J. Biol. Chem. 272:23165-23171 (1997).

67. G. Gimpl, U. Klein, H. Reilander, and F. Fahrenholz. Expression of the human oxytocin receptor in baculovirus-infected insect cells-high-affinity binding is induced by a cholesterol cyclodextrin complex. Biochemistry 34:13794-13801 (1995). 